\title{
LncRNA FTX promotes the malignant progression of colorectal cancer by regulating the miR-214-5p-JAG1 axis
}

\author{
Luxiang Pan ${ }^{1 \#}$, Mingrui Du ${ }^{2,3 \#}$, Haixia Liu ${ }^{4 \#}$, Boyang Cheng ${ }^{3}$, Maorong Zhu ${ }^{3}$, Bo Jia ${ }^{5}$, Yinwen Wang ${ }^{3}$, \\ Wei He ${ }^{1}$, Xiaoju Li ${ }^{1}$, Chenlin Liu ${ }^{1}$, Jintao Gu ${ }^{1}$, Meng Li ${ }^{1}$, Yingqi Zhang ${ }^{1}$, Li Yao ${ }^{6}$, Yi Zhang ${ }^{1}$, Qiang Hao ${ }^{1}$ \\ ${ }^{1}$ State Key Laboratory of Cancer Biology, Biotechnology Center, School of Pharmacy, Fourth Military Medical University, Xi'an, China; \\ ${ }^{2}$ Department of Orthopedics, Tangdu Hospital, Fourth Military Medical University, Xi'an, China; ${ }^{3}$ School of Basic Medicine, Fourth Military \\ Medical University, Xi'an, China; ${ }^{4}$ Department of Gynecology and Obstetrics, Shandong Provincial Hospital Affiliated to Shandong First Medical \\ University, Jinan, China; ${ }^{5}$ Department of Neurosurgery, Xijing Hospital, Fourth Military Medical University, Xi'an, China; ${ }^{6}$ Department of \\ Pathology, Xi'an No. 3 Hospital, The Affiliated Hospital of Northwest University, Xi'an, China \\ Contributions: (I) Conception and design: Q Hao, Y Zhang, L Yao; (II) Administrative support: Yin Zhang, M Li, J Gu; (III) Provision of study \\ materials or patients: L Pan, M Du, H Liu; (IV) Collection and assembly of data: B Cheng, M Zhu, Y Wang, W He; (V) Data analysis and \\ interpretation: X Li, C Liu, B Jia; (VI) Manuscript writing: All authors; (VII) Final approval of manuscript: All authors. \\ \#These authors contributed equally to this work. \\ Correspondence to: Qiang Hao. State Key Laboratory of Cancer Biology, Biotechnology Center, School of Pharmacy, Fourth Military Medical \\ University, Xi'an, China. Email: haosuq@fmmu.edu.cn; Yi Zhang. State Key Laboratory of Cancer Biology, Biotechnology Center, School of \\ Pharmacy, Fourth Military Medical University, Xi'an, China. Email: Zhangyi@fmmu.edu.cn; Li Yao. Department of Pathology, Xi'an No. 3 Hospital, \\ The Affiliated Hospital of Northwest University, Xi'an, China. Email: yaoli@fmmu.edu.cn.
}

Background: Long non-coding RNAs (lncRNAs) have recently been found to be vital regulators of various cancers, including colorectal cancer (CRC). It has been previously reported that the dysregulated expression of lncRNA Five prime to Xist (FTX) is involved in carcinogenesis. However, the role of lncRNA FTX in the progression of $\mathrm{CRC}$ is still unclear.

Methods: Fluorescence in situ hybridization (FISH) was used to detect the expression of lncRNA FTX and miR-214-5p in CRC tissues. Cell Counting Kit-8 assay, transwell assay, wound-healing assay, and proliferation assay were used to explore the function of lncRNA FTX in CRC cells. Quantitative real-time polymerase chain reaction (qRT-PCR), western blotting, and luciferase reporter assay were used to confirm the relationship between lncRNA FTX and miR-214-5p-jagged canonical Notch ligand 1 (JAG1). We further explored the role of lncRNA FTX in vivo using xenograft tumor assay.

Results: lncRNA FTX was found to be upregulated in CRC tissues by FISH. The downregulation of endogenous lncRNA FTX expression inhibited CRC cell proliferation, migration, and invasion. Mechanistically, lncRNA FTX sequestered miR-214-5p and thus released its repression on JAG1, driving the malignant progression of CRC.

Conclusions: These findings give rise to a new perspective, the lncRNA FTX-miR-214-5p-JAG1 regulatory axis, in exploring the cancer-promoting mechanism of lncRNA FTX in CRC.

Keywords: lncRNA FTX; miR-214-5p; progression; colorectal cancer (CRC)

Submitted May 27, 2021. Accepted for publication Jul 19, 2021.

doi: 10.21037/atm-21-2755

View this article at: https://dx.doi.org/10.21037/atm-21-2755

(c) Annals of Translational Medicine. All rights reserved. 


\section{Introduction}

Colorectal cancer (CRC) is 1 of the 3 most frequently diagnosed cancers and the fifth leading cause of cancerrelated mortality in women and men $(1,2)$. Aggressive surgical resection and chemotherapy are the mainstays of treatment for CRC, but the 5 -year survival rate for patients with advanced disease is less than $10 \%$ (3). The causative factors of CRC involve various influences, and the molecular mechanisms of tumor development are unclear (4). Therefore, it is essential to elucidate the molecular mechanisms of CRC carcinogenesis and development.

Long non-coding RNAs (lncRNAs) are a large class of transcripts comprising more than 200 bases, which do not have the potential to code protein (5). LncRNAs are crucial for cell proliferation, homeostasis, and differentiation $(6,7)$, and their expression is considered to be distorted in cancerous cells. Previous studies have reported that lncRNAs are related to a variety of malignant tumors (8). Biological research on non-coding RNAs and gene therapy strategies for lncRNAs in CRC has recently attracted much attention $(9,10)$.

The five prime to Xist (FTX) gene is a non-proteincoding gene on the human $\mathrm{X}$ chromosome and a conserved transcript located in $\mathrm{X}$ chromosome inactivation (XCI) center (11). In addition to encoding 2 miRNA molecules, $F T X$ also encodes a highly conserved transcription product, lncRNA FTX (12). In a previously published report, the essential role of IncRNA FTX in the regulation of Xist expression was demonstrated (13). Recent studies have revealed that lncRNA FTX may affect tumor genesis and development through regulating miRNAs $(14,15)$ and that it can act as a competing endogenous RNA to interact with miRNAs $(16,17)$. For example, in gastric cancer, lncRNA FTX has been found to promote tumor progression by targeting miR-215 (18), and lncRNA FTX knockdown suppresses the proliferation, migration, and invasion of renal cancer cells (19). Until now, the role of abnormal expression of lncRNA FTX in cancer, including CRC, remains largely unknown.

In the present study, we found that lncRNA FTX was upregulated in CRC compared with adjacent normal tissues. We also found that CRC cell proliferation, invasion, migration, and tumorigenicity were restricted after lncRNA FTX knockdown in vivo and in vitro. After antagonizing the activity of miR-214-5p, the inhibition of tumor malignant biological properties nearly disappeared. Therefore, we investigated whether lncRNA FTX might play the role of sequestering miR-214-5p from normal target jagged canonical Notch ligand 1 (JAG1). We present the following article in accordance with the ARRIVE reporting checklist (available at https://dx.doi.org/10.21037/atm-21-2755).

\section{Methods}

\section{Cell culture conditions and cell lines}

CRC cell lines SW620 and HCT116 were obtained from the China Academia Sinica Cell Repository (Shanghai, China), which were derived from a male donor. The karyotypes can be found in the research by Camps et al. (20). Fetal bovine serum (10\%) and 50 units/mL penicillin/ streptomycin (Gibco, CA, USA) were added to McCoy's 5A Medium (Gibco, USA) and Dulbecco's Modified Eagle Medium (Gibco, USA), which were respectively used to culture the HCT-116 and SW-620 cells. All cell lines were cultured in a sterile incubator at $37{ }^{\circ} \mathrm{C}$ in a humidified atmosphere containing $5 \% \mathrm{CO}_{2}$.

\section{Patients and clinical samples}

In total, 17 CRC tissue specimens were collected from patients, including 5 women and 12 men who had not received radiation or chemotherapy and who underwent surgery at Xijing Hospital of the Fourth Military Medical University (FMMU; Shaanxi, China). The pathology of every sample was validated by 2 professional and independent pathologists. General clinical information was collected. The study was conducted in accordance with the Declaration of Helsinki (as revised in 2013). The study was approved by the Clinical Research Ethics Committee of the Fourth Military Medical University (FMMU) (No.: 202003-183). Before enrollment in the study, written informed consent was collected from all patients.

\section{$R N A$ extraction and quantitative real-time polymerase chain reaction ( $q R T-P C R)$}

TRIzol reagent (Invitrogen, CA, USA) was used to isolate the total RNA from CRC cells. In total, $1 \mu \mathrm{g}$ total mRNA was used to compose cDNA with a kit (Promega, WI, USA) for reverse transcription-polymerase chain reaction (RTPCR) (21); qRT-PCR was performed in $20 \mu \mathrm{L}$ volumes with SYBR Green reagents (Takara, Japan) using the Applied Biosystems 7500 Fast System (Applied Biosystems, USA). The instrument parameters were set as follows: 1 cycle 
with a pre-incubation step $\left(95^{\circ} \mathrm{C}\right.$ for $\left.2 \mathrm{~min}\right)$ and 40 cycles with an amplification step $\left(95^{\circ} \mathrm{C}\right.$ for $15 \mathrm{~s}$ and $60{ }^{\circ} \mathrm{C}$ for $60 \mathrm{~s}$ ), followed by a melting step $\left(95^{\circ} \mathrm{C}\right.$ for $15 \mathrm{~s}, 60^{\circ} \mathrm{C}$ for $1 \mathrm{~min}$, and then $95^{\circ} \mathrm{C}$ continuous) and a cooling step of $1 \mathrm{~s}$ at $40{ }^{\circ} \mathrm{C}$. The relative expression of mRNA was normalized to $\beta$-actin and quantified with the $2^{-\Delta \mathrm{Ct}}$ method. All qRT-PCR experiments were conducted 3 times. U6 and miR-214-5p primers were provided by RiboBio (Guangzhou, China). The remaining primer sequences were as follows:

* IncRNA FTX forward: 5'- GAGGATGAATTACGGTTGCC -3'

* IncRNA FTX reverse: 5'- CCTGAGATACCTGTTCCTTTGT -3'

* $\beta$-actin forward: 5'-CCACGAAACTACCTTCAACTCC-3'

* $\beta$-actin reverse: 5'-TCATACTCCTGCTGCTTGCTGATC-3'

* JAG1 forward: 5'- ATTACCAGGATAACTGTGCGAA-3'

* JAG1 reverse: 5'- CAAATGTGCTCCGTAGTAAGAC-3'

\section{Western blotting}

The same quantities of denatured protein samples were divided on a separated using $10 \%$ sodium dodecylsulfatepolyacrylamide gel electrophoresis and then placed on a polyvinylidene difluoride membrane. Then, $5 \%$ skimmed milk powder dissolved in Tris-buffered saline (TBS)/0.05\% Tween-20 was used to block the membranes. The cells were then incubated overnight at $4{ }^{\circ} \mathrm{C}$. Anti-JAG1 $(1: 1,000$; Abcam, USA) and $\beta$-actin (1:5,000; CWBIO, Wuhan, China) primary antibodies and dilutions were used according to manufacturers' instructions. Membranes were incubated at room temperature with goat anti-mouse IRDye $800 \mathrm{CW}$ secondary antibody or goat anti-rabbit IRDye 800CW for $1 \mathrm{~h}$. The Odyssey CLx Imaging System (LI-COR, Lincoln, NE, USA) was used to scan and analyze the images.

\section{Cell viability assay}

Each well on a 96-well plate was seeded with 5,000 cells in $100 \mu \mathrm{L}$ of complete culture medium. After $48 \mathrm{~h}$, each well was supplemented with $10 \mu \mathrm{L}$ Cell Counting Kit-8 (CCK-8) reagent and then incubated for $2 \mathrm{~h}$ at $37^{\circ} \mathrm{C}$. Using a plate reader for measurement (Bio-Rad, USA), optical density (OD) at $450 \mathrm{~nm}$ was used to establish the rate of cell growth.

\section{Cell migration and invasion assay}

A total of $5 \times 10^{5}$ cells were added to each well in a 6-well plate and cultured for $24 \mathrm{~h}$. The confluent cell monolayer was damaged after scraping it with the tip of a $200 \mu \mathrm{L}$ pipette. A serum-free medium was added for cell culture. Observing the healing of scratches at 0 and $48 \mathrm{~h}$, the wells were then photographed using a microscope. Image-Pro Plus 6.0 software was used to measure the distances.

Cells were retrieved and resuspended in an incomplete medium. A total of $1 \times 10^{5}$ cells were then added into a transwell chamber (Corning, USA) preloaded with Matrigel (BD Bioscience, USA). The chamber was placed onto a 24-well plate with a complete medium containing $20 \%$ serum. After incubation for $48 \mathrm{~h}$, the chamber on top was removed, and the cells remaining on the surface of the upper membrane were wiped off with cotton buds. After air drying, cells on the exterior of the lower membrane were fixated with $4 \%$ paraformaldehyde for $20 \mathrm{~min}$, colored with $0.1 \%$ crystal violet, and washed 3 times before air drying. Cells were then counting using a light microscope.

\section{Luciferase reporter assay}

The sequence of wild-type (WT) and mutant-type (MUT) lncRNA FTX and mRNA 3 '-untranslated region (3'-UTR) of JAG were synthesized. HCT-116 cells were then added to 24-well plates. After $24 \mathrm{~h}$, cells pretreated with miR214-5p inhibitor or pretreated mimics were transfected with reporter plasmids. Untreated CRC cells were also transfected. Luciferase activity was analyzed after $24 \mathrm{~h}$ in the cells. Relative light units, determined by Renilla luciferase assay, were divided by the value measured by firefly luciferase, and then the relative luciferase values were obtained.

\section{Lentiviral, plasmid, and miRNA mimics package and cell transfection}

The loss- and gain-of-function experiments were conducted by infecting HCT-116 and SW620 cells with sh-FTX or shNegative contril (shNC) lentivirus, which were obtained from GeneChem (Shanghai, China), at a multiplicity of infection of 10 with $8 \mathrm{~g} / \mathrm{mL}$ polybrene (GeneChem, China). Four days after infection, puromycin-resistant cell lines were screened using a medium containing $2 \mathrm{~g} / \mathrm{mL}$ puromycin (Thermo Fisher, USA).

Transfection of human miR-214-5p mimic, miRNA 
negative control, and miR-214-5p inhibitor, synthesized by RiboBio (Guangzhou, China), was conducted with Lipofectamine 3000 Reagent (Invitrogen, USA) for $15 \mathrm{~min}$ at room temperature. The mixture was then added to the cell supernatant with a culture medium and incubated in a cell incubator for 2 days before it completely entered the cells.

\section{Fluorescence in situ bybridization (FISH) assay}

After fixation for $6 \mathrm{~h}$ in $4 \%$ paraformaldehyde, the CRC tissues were dried in a graded ethanol series and immersed in paraffin. The embedded tissues were sliced into sections and then incubated for $2 \mathrm{~h}$ at $62{ }^{\circ} \mathrm{C}$. Next, the sections were treated according to the following sequence: $15 \mathrm{~min}$ with dimethylbenzene xylene, $5 \mathrm{~min}$ in anhydrous ethanol, $5 \mathrm{~min}$ in $85 \%$ alcohol, and $5 \mathrm{~min}$ with $75 \%$ alcohol. The tissue sections were then mounted onto slides and treated for $30 \mathrm{~min}$ at $37^{\circ} \mathrm{C}$ with proteinase $\mathrm{K}(2 \mu \mathrm{g} / \mathrm{mL}$; Servicebio, China) and $3 \% \mathrm{H}_{2} \mathrm{O}_{2}$, followed by washing, prehybridization for $1 \mathrm{~h}$ at $37^{\circ} \mathrm{C}$, and hybridization (RiboBio, China) with $1 \mu \mathrm{L}$ of hybrid solution at $46^{\circ} \mathrm{C}$ overnight, which contained miR-214-5p and lncRNA FTX probes. The biotin-labeled probe (the sequence of IncRNA FTX was 5'-CT+TCGT CAACACGGCACAA+TCTACAGCATAGT+TTCTG AT+TGAGTGTGGCGCAACCGTAAT+TCAT+TC GCCCTC-3') was designed by GenePharma (Shanghai, China) and used the streptavidin-biotin system to design to detect lncRNA specifically. The sequence of miR-214-5p was 5'-GCACAGCAAGTGTAGACAGGCA-3' and was designed by Servicebio. Finally, the fluorescence signal was observed under a microscope and processing fluorescence signal strength by ImageJ software.

\section{Immunobistochemical (IHC) analysis}

Formalin and paraffin were used to fix and embed the collected tissues, respectively. The slices were dewaxed in xylene and rehydrated in alcohol. Following the blockage of non-specific antigen-binding at $37{ }^{\circ} \mathrm{C}$ with $5 \%$ bovine serum albumin (BSA) for $1 \mathrm{~h}$, antibodies against Ki67 (1:200; Abcam, USA), vimentin (1:1,000; Abcam, USA), MMP3 (1:1,000; Abcam, USA), and JAG1 (1:200; Abcam, USA) were added to the slices, and the slices incubated overnight at $4{ }^{\circ} \mathrm{C}$. The slices were then hybridized with horseradish peroxidase-labeled secondary antibody for $1 \mathrm{~h}$ at $37^{\circ} \mathrm{C}$. Diaminobenzidine was used to stain the samples, and hematoxylin was used to counterstain them. Representative images were observed under a microscope (IX-71; Olympus, Japan).

\section{In vivo tumorigenesis assay}

Animal experiments were performed under a project license (No.:202003-183) granted by the Institutional Animal Care and Use Committee of FMMU, in compliance with institutional guidelines for the care and use of animals. The animals were reared at the Experimental Animal Center of FMMU. We screened HCT-116 cells with stably silenced lncRNA FTX using puromycin. We used the subcutaneous xenograft mouse model to evaluate the ability of tumor formation. First, $200 \mu \mathrm{L}$ phosphate-buffered saline was used to suspend $5 \times 10^{6}$ HCT-116 cells, subcutaneously injected into BALB/c nude mice's right flanks (single factor experiment, $n=6$ per group). The miR-214-5p inhibitor and NC inhibitor used were antagomir (RiboBio, China), injected into the caudal vein. The injection volume for each nude mouse was $100 \mathrm{nmol}$, and the injection was administered twice per week. Digital calipers were used to measure the tumor dimensions every 3 days. After 4 weeks, the tumors were removed, fixed in neutral formalin, and embedded in paraffin.

\section{Statistical analysis}

GraphPad Prism version 8.0 was used to perform the statistical analysis. $\mathrm{P}<0.05$ indicated statistical significance. The student's $t$-test was used for the 2-group comparison. Carcinoma and adjacent tissue samples from the same patient were tested by paired $t$-test, and the rest were tested by unpaired $t$-test. One-way analysis of variance was used to compare differences between 3 or more groups. All quantitative data were expressed as mean \pm standard error of the mean $(\mathrm{SEM})(\mathrm{n}<60)$ or mean \pm standard deviation $(\mathrm{SD})$ $(n>60)$.

\section{Results}

\section{Expression and location of IncRNA FTX in CRC tissues and cells}

The expression of lncRNA FTX was significantly higher in CRC tissues compared with normal colon tissues based on data from Sangerbox (http://sangerbox.com/), ENCORI (http://starbase.sysu.edu.cn/panCancer.php) and Gene Expression Profiling Interactive Analysis (http://gepia. 
cancer-pku.cn/) (Figure $1 A$ and Figure S1A,S1B). KaplanMeier analysis indicated that a high expression of lncRNA $F T X$ was associated with poor disease-free survival in 269 CRC patients $(\mathrm{P}=0.0026)$ (Figure 1B). To confirm lncRNA FTX expression in CRC tissues, we performed FISH in a tissue microarray containing 17 pairs of CRC tissues and surrounding non-cancerous tissues and found that FTX had higher expression in tumor tissues (Figure 1C,1D). To further investigate the confusing location of lncRNA FTX expression, we detected the localization of lncRNA FTX in CRC cells by FISH assay. The results showed that lncRNA FTX was distributed in the CRC cell nucleus and cytoplasm (Figure 1E). Considering the location of lncRNA $F T X$ on the $\mathrm{X}$ chromosome, we detected the expression of lncRNA FTX in different gender donor samples. We found that there was no significant difference in the expression of lncRNA FTX in CRC samples from male and female donors (Figure S1C). Therefore, lncRNA FTX was located in both the nucleus and cytoplasm and was found to be highly expressed in CRC tissues and cells.

\section{Knockdown of lncRNA FTX suppressed the proliferation, migration, and invasion of CRC cells}

To evaluate the function of IncRNA FTX in CRC cells, we constructed HCT-116 and SW620 cells, which stably expressed low lncRNA FTX basal level by lentivirus infection. On transduction of $3 F T X$ shRNAs, we found that sh-FTX 2 could effectively downregulate lncRNA FTX expression by approximately 41-57\% (Figure S1D,S1E). CCK-8 assay demonstrated that lncRNA FTX knockdown significantly suppressed the proliferation of HCT-116 and SW620 cells (Figure 2A). Moreover, immunofluorescence (IF) assay confirmed that low levels of lncRNA FTX reduced Ki67 expression (Figure 2B). In addition, we conducted transwell and wound-healing assays. The results indicated that lncRNA FTX depletion significantly curtailed the migration ability of both HCT-116 cells and SW620 cells (Figure 2C,2D), and suppressed CRC cell invasion (Figure 2E). These results confirmed that lncRNA FTX knockdown could reduce the proliferation, invasion, and migration of CRC cells in vitro.

\section{LncRNA FTX sequester miR-214-5p in CRC}

Although early reports indicated that IncRNA FTX might be located in the nucleus, follow-up reports have demonstrated that lncRNA FTX is localized in both the nucleus and the cytoplasm $(22,23)$. Our results indicated that lncRNA FTX is located in the nucleus and cytoplasm of CRC cells, making it possible to interact with miRNA. Using a non-coding RNA database (starBase), we selected miR-214-5p as an alternative target of lncRNA FTX, as it has the most binding sites and rates highest on multiple prediction sites (Figure S1F). MiR-214-5p works as a tumor inhibitor in different cancers and is downregulated in CRC patients (Figure S1G) $(24,25)$. To further verify the interaction between lncRNA FTX and miR-214-5p, a tissue chip was used to detect miR-214-5p in a previously assessed tissue microarray. As shown in Figure 3A,3B, miR-214-5p was notably downregulated in CRC tissues compared with adjacent peritumoral tissues.

Interestingly, lncRNA FTX expression had a negative correlation with that of miR-214-5p (Figure 3C). Therefore, we subcloned MUT and WT miR-214-5p binding sites of lncRNA FTX into dual-luciferase reporter vectors to confirm whether lncRNA FTX could regulate miR-214$5 \mathrm{p}$ (Figure 3D). The luciferase assay demonstrated that the miR-214-5p mimics downregulated the relative luciferase activity of lncRNA FTX WT-treated CRC cells but did not impact that of lncRNA FTX MUT-treated CRC cells (Figure $3 E$ ). The expression of miR-214-5p was upregulated with the presence of lncRNA FTX knockdown (Figure 3F). These findings indicate that IncRNA FTX may sequester miR-214-5p from its normal targets in CRC cells.

\section{LncRNA FTX affects cell proliferation, migration, and invasion of CRC cells through the regulation of miR-214-5p}

To confirm the involvement of miR-214-5p in lncRNA FTX-mediated tumor-promoting activity, rescue experiments were conducted to evaluate the impact of lncRNA FTX and miR-214-5p on CRC cells. CCK-8 assay demonstrated that the suppression of cell growth induced by the knockdown of IncRNA FTX was alleviated by inhibiting miR-214-5p in HCT-116 and SW620 cells (Figure 4A). Low levels of Ki67 expression, as determined by IF, also showed that low levels of IncRNA FTX reduced cell proliferation. In contrast, the inhibition of miR-214$5 \mathrm{p}$ had the opposite effect on cell proliferation (Figure $4 B$ ). The wound-healing assay demonstrated that lncRNA FTX knockdown significantly suppressed the migration of cells, whereas the restrain miR-214-5p abolished these effects (Figure $4 C, 4 D$ ). Similarly, the invasive capacity of HCT116 and SW620 cells was significantly suppressed with miR-214-5p inhibition (Figure 4E). These findings 
A
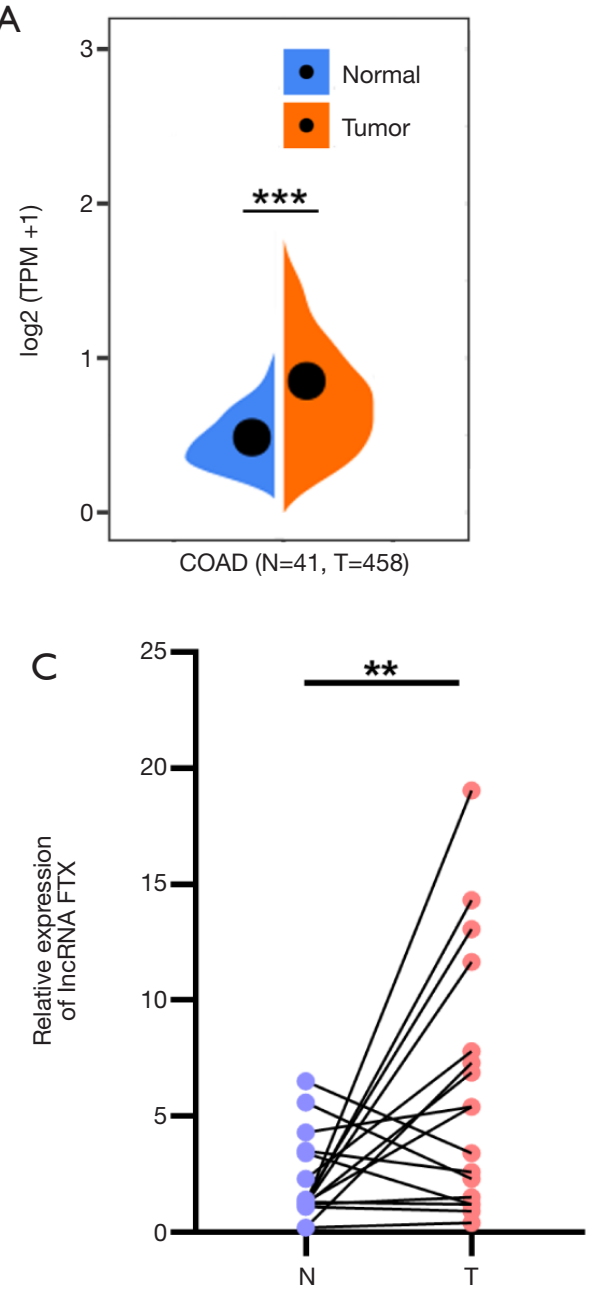

E

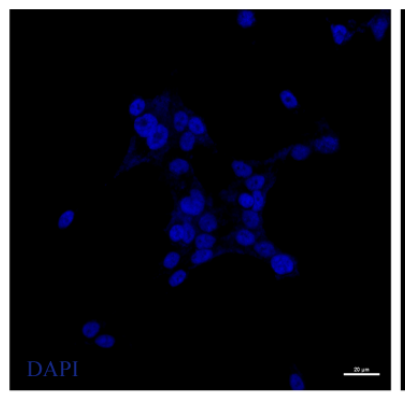

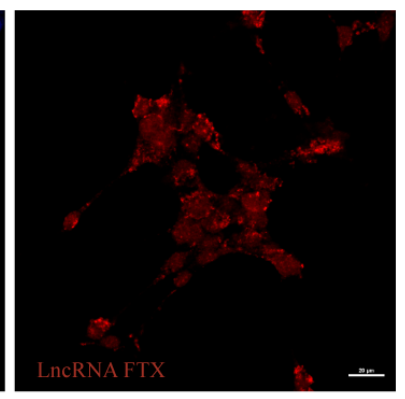

B

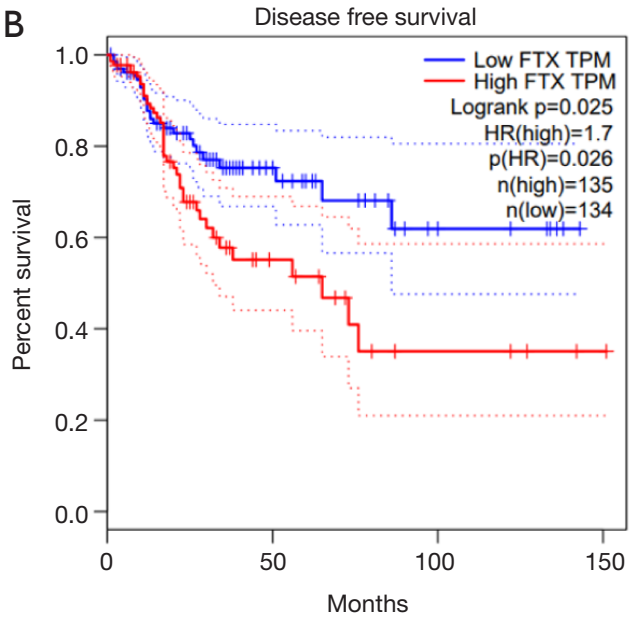

D
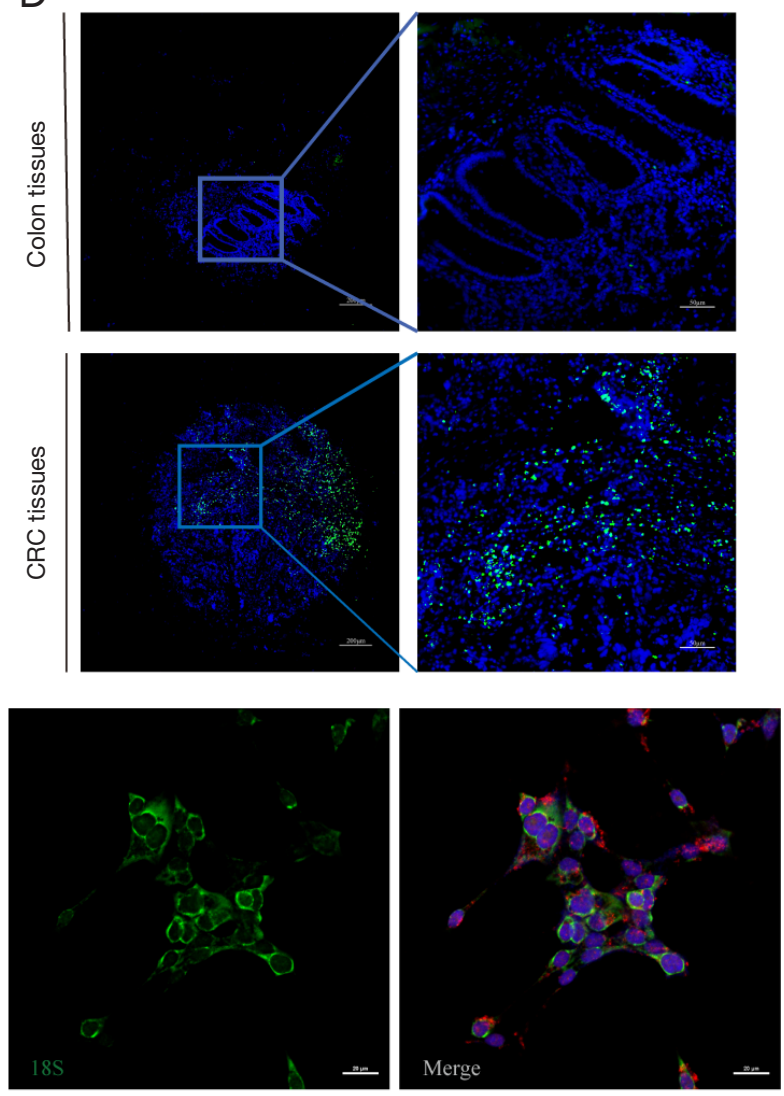

HCT116

Figure 1 Expression and location of LncRNA FTX in CRC tissues and cells. (A) Differences in LncRNA FTX expression between 458 CRC tissues and 41 normal colon tissues (data from Sangerbox). (B) Kaplan-Meier analysis showing the correlation between lncRNA FTX expression and disease-free survival of CRC patients $(\mathrm{n}=269)$. (C) LncRNA FTX relative expression in CRC and non-cancerous tissues in 17 paired patients. P value was established by paired $t$-test. (D) LncRNA FTX (green) in CRC and non-cancerous tissues was established by FISH. Nuclei stained with 4',6-diamidino-2-phenylindole (DAPI) are shown in blue. Magnification: 100× and 400×. (E) Location of lncRNA FTX (Red) was detected by FISH assay in CRC cells. Cytoplasmic marker $18 \mathrm{~S}$ is stained green. Magnification: $1,200 \times .{ }^{* *}, \mathrm{P}<0.01$; ***, $\mathrm{P}<0.001$. CRC, colorectal cancer. 

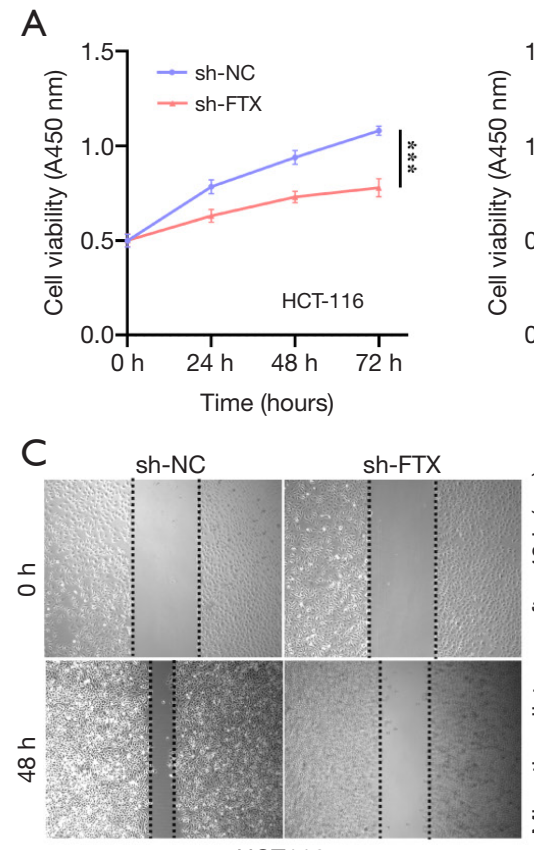

HCT116
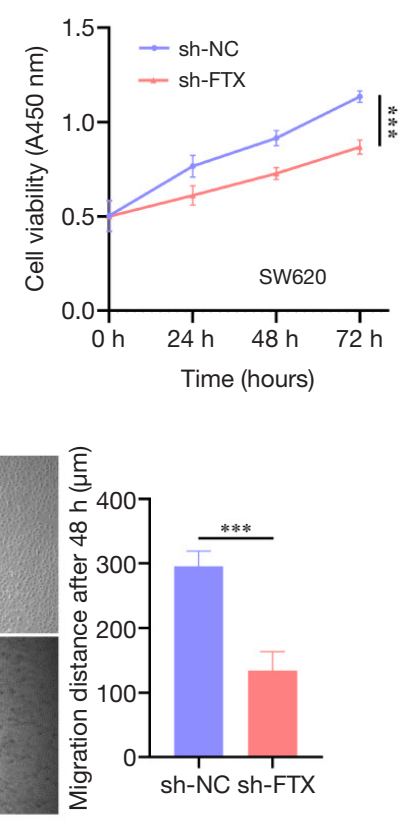

sh-FTX
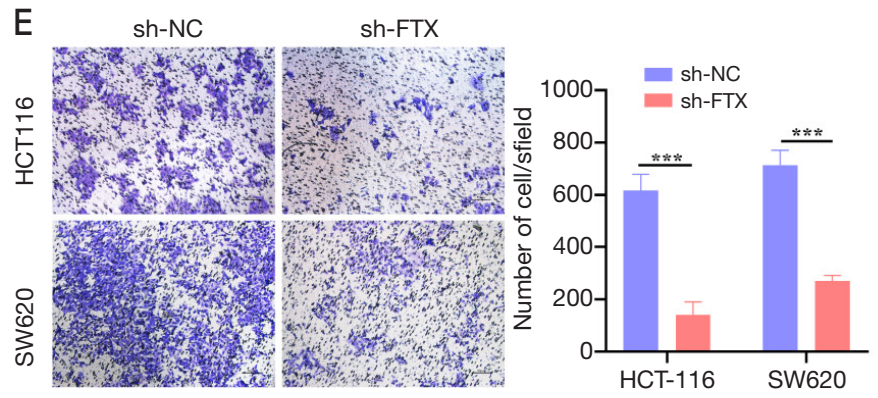

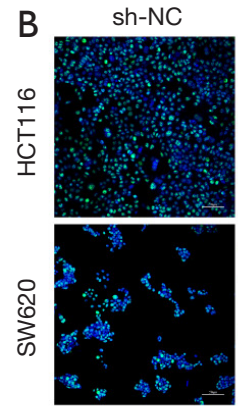

sh-FTX
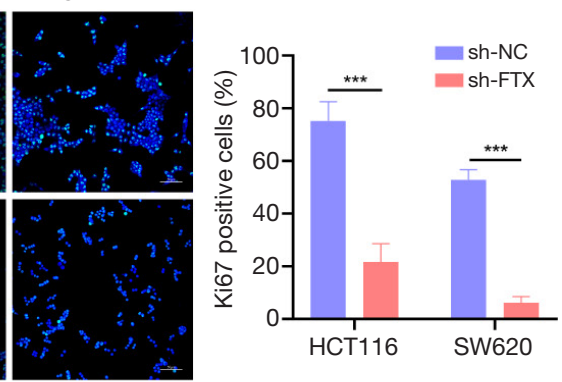

D
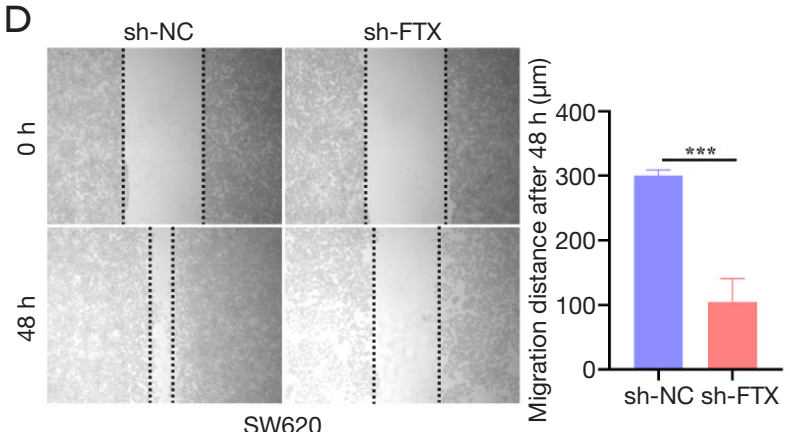

Figure 2 LncRNA FTX knockdown suppresses the proliferation, migration, and invasion of CRC cells. (A) Cell Counting Kit-8 was used to examine the effects of knocking down lncRNA FTX on the proliferation of HCT-116 and SW620 cells. (B) Representative immunofluorescence images and the fraction of cells expressing Ki67 (Ki67+ cells/DAPI-stained cells) after lncRNA FTX knockdown in CRC cells. Magnification: 100×. (C,D) Representative images of wound-healing assays used in the evaluation of the motility of CRC cells transfected with shNC or sh-FTX. (E) Representative images of transwell invasion assays used in the evaluation of the motility of CRC cells transfected with shNC or sh-FTX, stained with $0.5 \%$ crystal violet $(100 \times)$. ${ }^{* * *}, \mathrm{P}<0.001$. CRC, colorectal cancer.

demonstrated that lncRNA FTX affects cell migration, invasion, and proliferation of CRC cells through miR-214$5 \mathrm{p}$ in CRC cells.

\section{LncRNA FTX regulates the expression of $\mathcal{A} A G 1$ via miR- 214-5p}

To determine the effects of the lncRNA FTX-miR214-5p axis and its downstream pathways, we used TargetScanHuman (http://www.targetscan.org) and miRBase (https://www.mirbase.org) to predict that JAG1 might be a potential target of miR-214-5p. JAG1 has key roles in tumor progression (26). Data from Sangerbox indicate that JAG1 expression is significantly different in carcinoma and paracancerous tissues in CRC patients (Figure 5A). Therefore, we selected JAG1 as the potential target of miR-214-5p. To test the above hypothesis, we examined its expression in miR-214-5p-overexpressing CRC cells using qRT-PCR. The results indicated that JAG1 expression significantly decreased with miR-214$5 \mathrm{p}$ overexpression compared to cells expressing the NC mimic empty vector (Figure 5B). Therefore, JAG1 was 
A

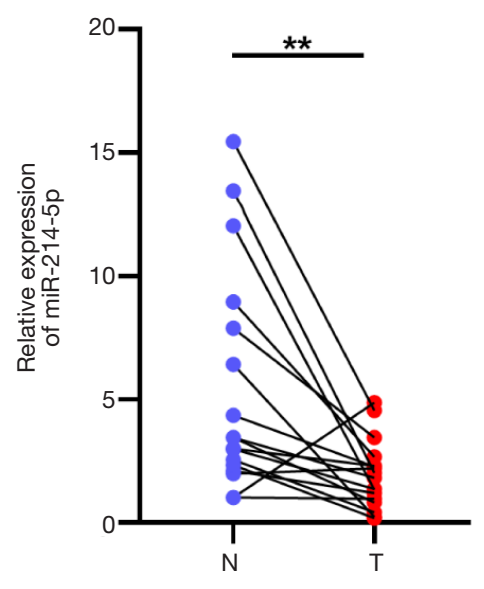

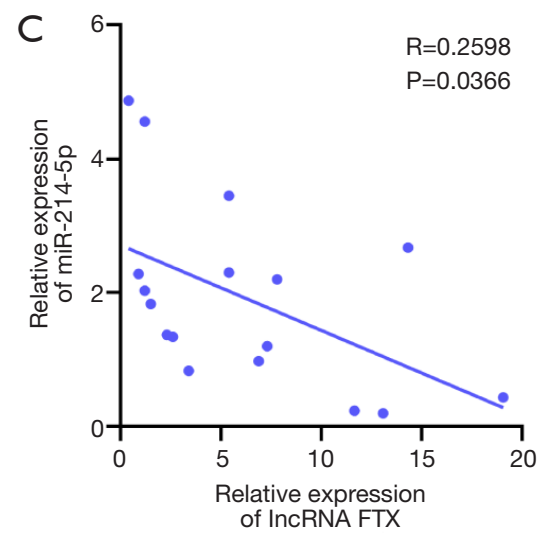

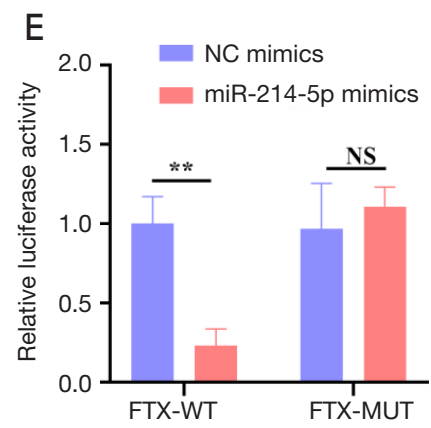

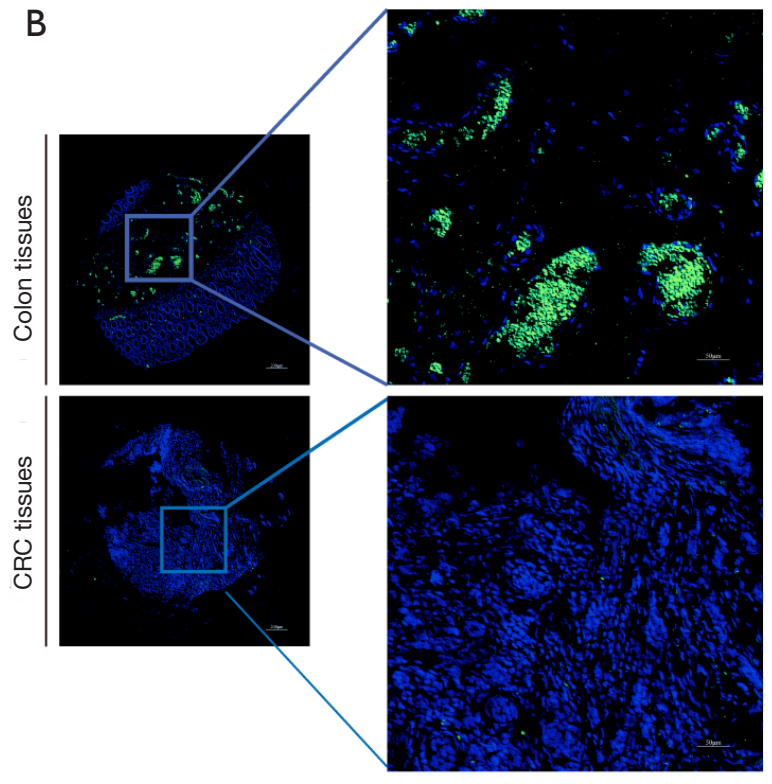

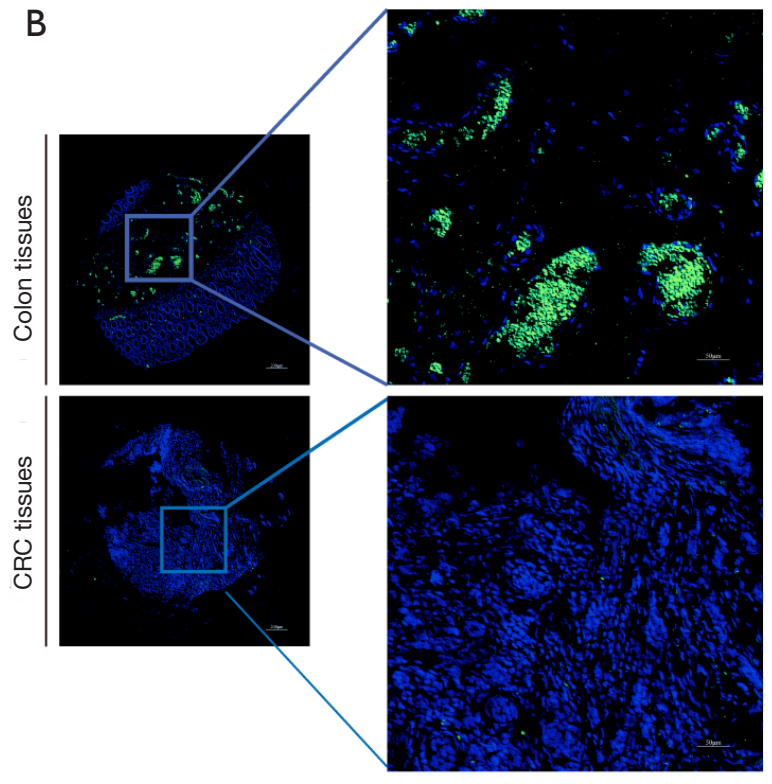

D
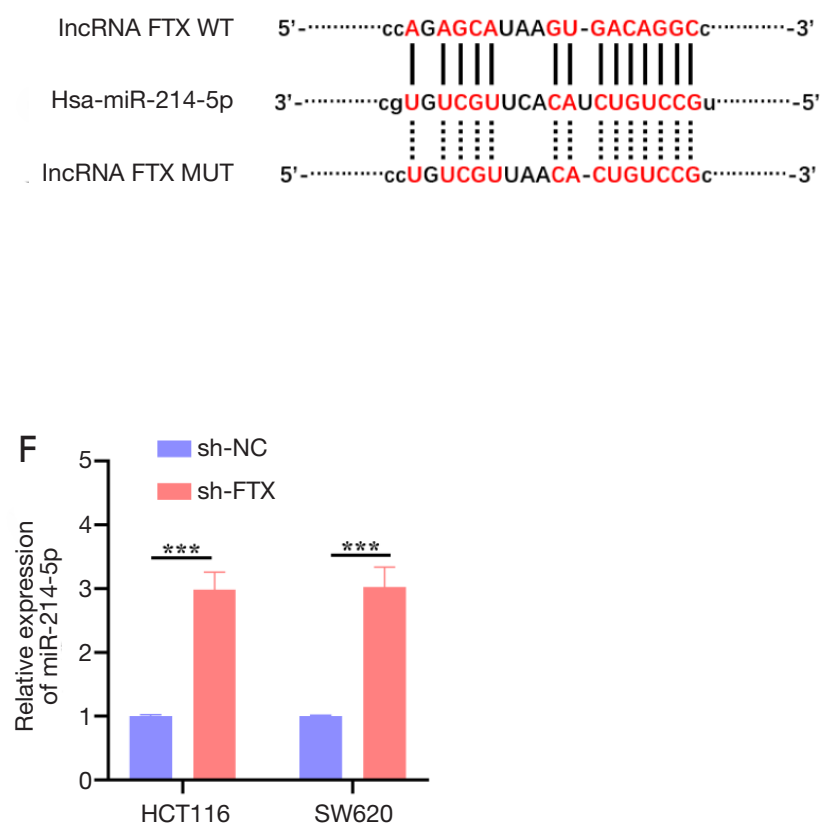

Figure 3 LncRNA FTX sequester miR-214-5p in CRC. (A) Relative expression level of miR-214-5p in CRC and non-cancerous tissues in 17 paired patients was analyzed. $\mathrm{P}$ value was established by paired $t$-test. (B) Representative images of miR-214-5p (green) in CRC and noncancerous tissues were established by fluorescence in situ hybridization. Nuclei stained with DAPI are shown in blue. Magnification: $100 \times$ and 400×. (C) Association between miR-214-5p expression and lncRNA FTX in CRC tissue specimens $\left(\mathrm{r}^{2}=0.2598, \mathrm{P}<0.05\right)$. To evaluate significance, Pearson's coefficient tests were conducted. (D) Potential binding sites of the miR-214-5p and lncRNA FTX. (E) Reporter plasmids incorporated with mutant or wild-type lncRNA FTX and miR-214-5p mimics were co-transfected into HCT-116 cells. After 48 h, luciferase activity was evaluated. (F) Quantitative real-time polymerase chain reaction assays identified the impact of miR-214-5p on lncRNA FTX expression. ** $\mathrm{P}<0.01$; ${ }^{* * *}, \mathrm{P}<0.001$; NS, nonsignificant. CRC, colorectal cancer. 


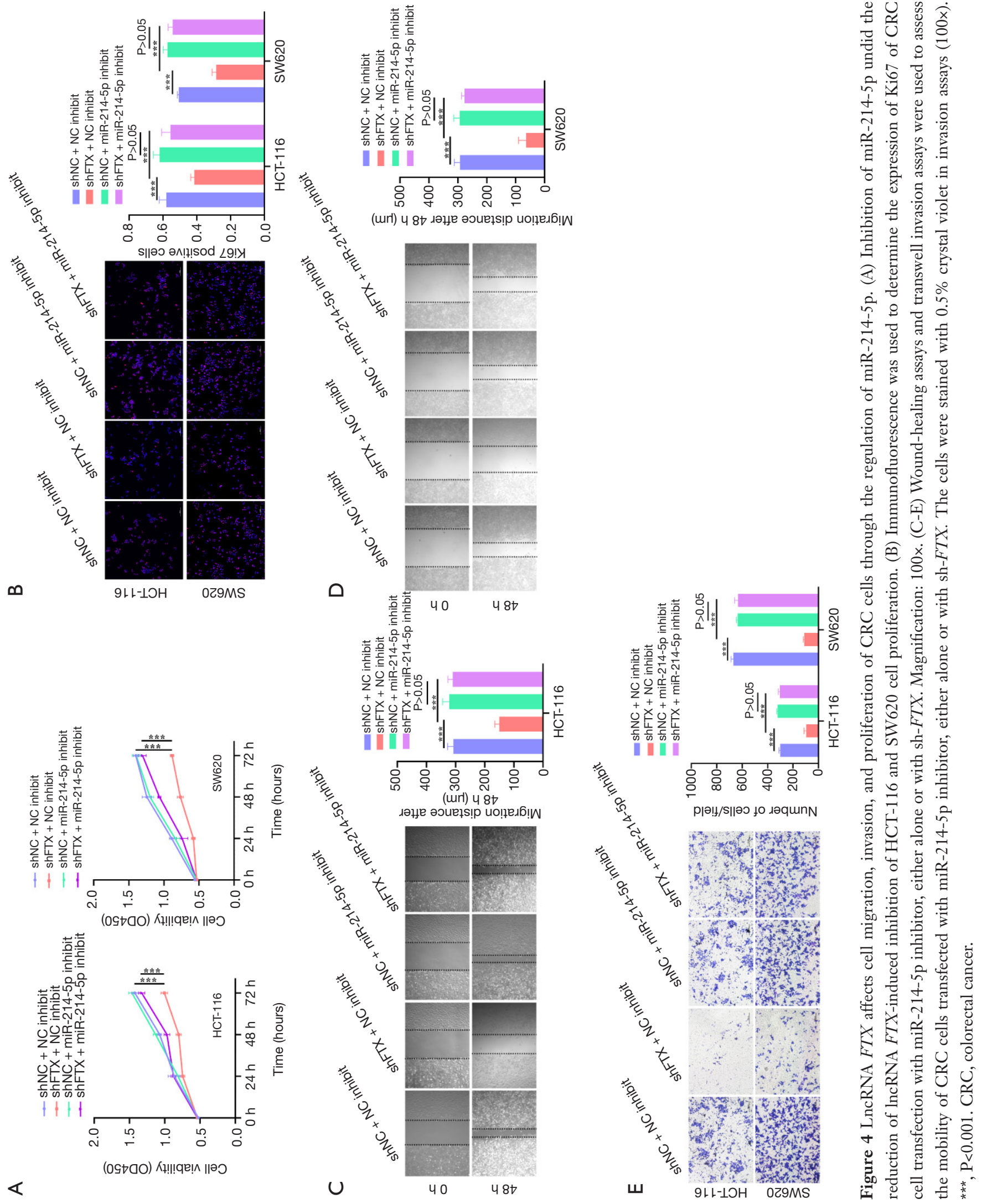




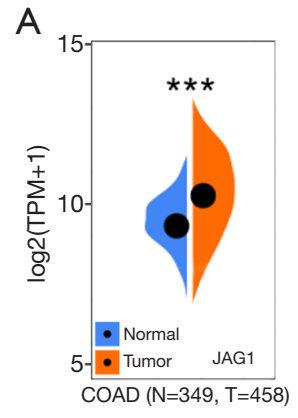

C

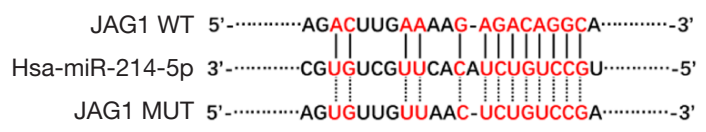

$\mathrm{E}$

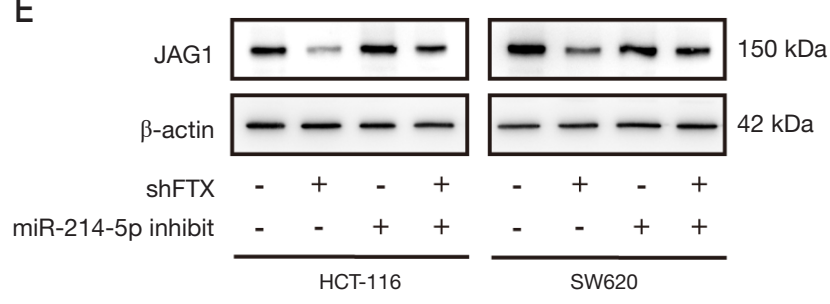

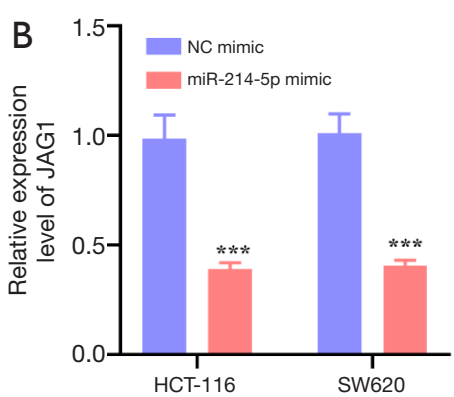
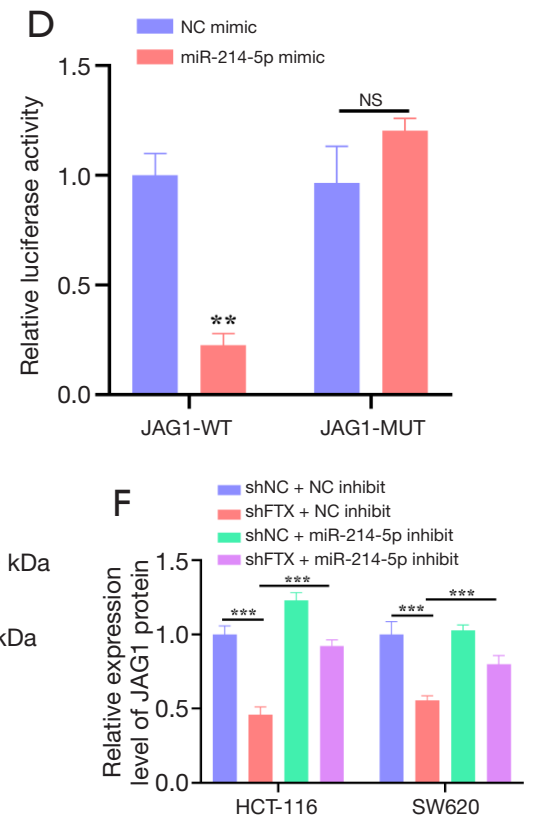

Figure 5 LncRNA FTX regulates JAG1 expression via miR-214-5p. (A) JAG1 expression in carcinoma and adjacent tissue of CRC patients. (B) Quantitative real-time polymerase chain reaction assays revealed the impact of miR-214-5p on JAG1 protein expression. (C) Predicted binding sequence of miR-214-5p was identified on JAG1 mRNA. (D) HCT-116 cells were co-transfected with reporter plasmids (JAG1 wild type and JAG1 mutant) and miR-214-5p mimics, and luciferase activity was measured. (E) Cells with sh-FTX were additionally treated with miR-214-5p inhibitor or NC inhibitor. Western blot assays were used to determine the JAG1 protein expression levels in SW620 and HCT116 cells. (F) Statistical data of cells with sh-FTX additionally treated with miR-214-5p inhibitor or $\mathrm{NC}$ inhibitor. **, $\mathrm{P}<0.01$; *** $\mathrm{P}<0.001$; NS, nonsignificant. CRC, colorectal cancer.

chosen as the assumed target of miR-214-5p in additional observations. We constructed JAG1 full 3'-UTRs that contained the binding site of miR-214-5p that was predicted and a mutation of the site (Figure 5C). The overexpression of miR-214-5p mimic reduced the luciferase activity of WT JAG1 reporters, but not the MUT reporter, as shown by luciferase assay (Figure 5D), indicating that JAG1 was the direct target of miR-214-5p. Furthermore, JAG1 protein levels reduced on lncRNA FTX knockdown, whereas during the rescue experiments, the miR-214-5p inhibitor could partially prevent reduction in JAG1 levels in CRC cells
(Figure 5E, $5 F$ ). These results indicated that JAG1 was the target of miR-214-5p in CRC and that lncRNA FTX had a functional role in regulating JAG1 expression by targeting miR-214-5p.

\section{LncRNA FTX knockdown inbibits CRC growth in vivo}

The findings indicated that lncRNA FTX affects cell migration, invasion, and proliferation of CRC cells via the regulation of miR-214-5p. To determine the function of lncRNA FTX and miR-214-5p in tumor growth 

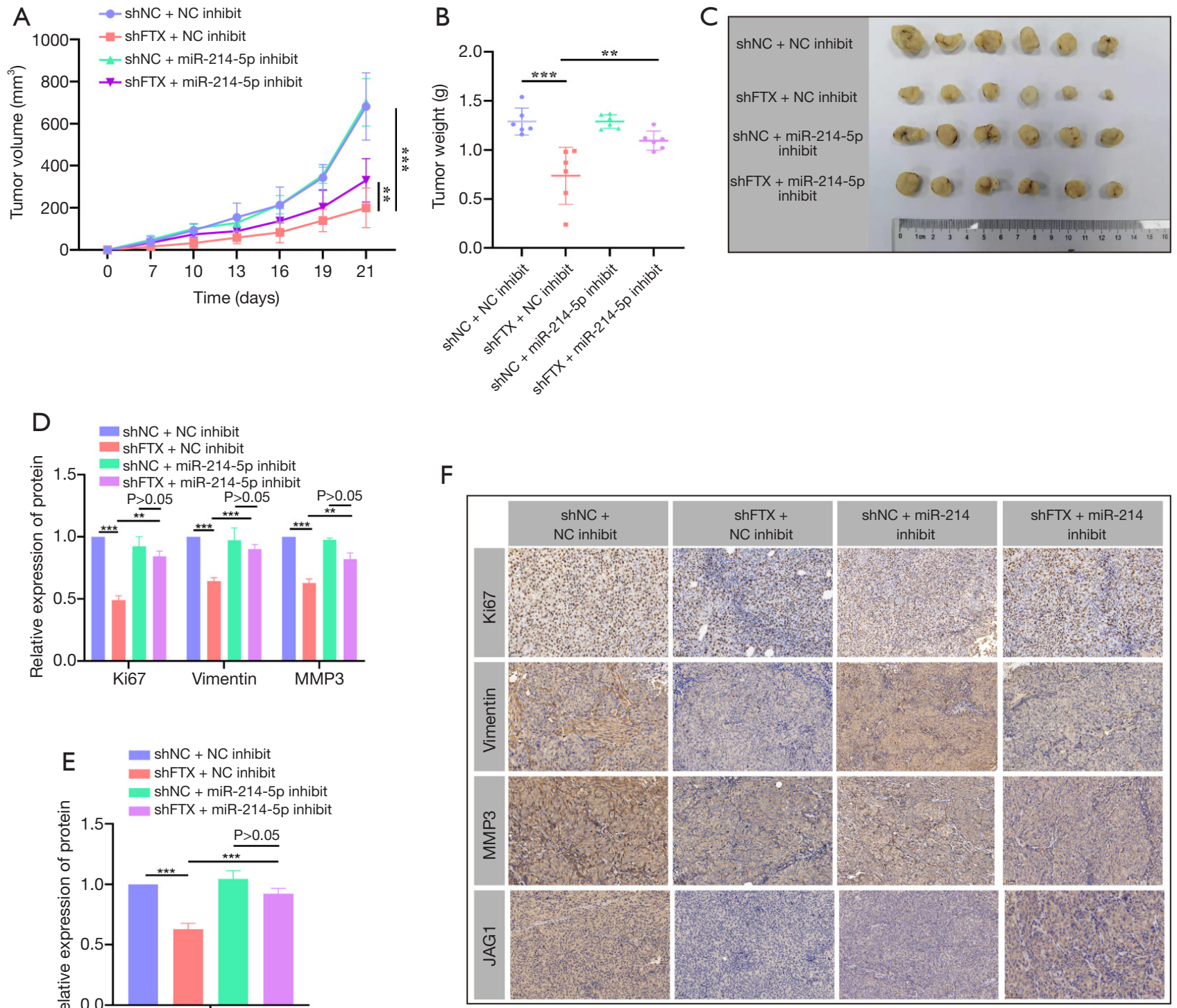

Figure 6 LncRNA FTX knockdown inhibits the growth of CRC in vivo. (A) Every 3 days, tumor volume (n=6) was measured. (B) Average weight of tumors resected from mice $(n=6)$. (C) Pictures of the tumors from the mice at 21 days following injection with the cells as indicated. (D,E) Quantification of Ki67-, vimentin-, MMP3-, and JAG1-positive CRC cells in xenograft tumors excised from mice. (F) Representative immunohistochemical images of Ki67, vimentin, MMP3, and JAG1 expression. Magnification: 400×. Data are presented as the mean \pm standard error of mean. P-value was established by unpaired $t$-test. ${ }^{* *}, \mathrm{P}<0.01 ;{ }^{* * *}, \mathrm{P}<0.001$. CRC, colorectal cancer.

in vivo, sh-FTX HCT-116 cells were subcutaneously inoculated into nude mice. During the predetermined time following subcutaneous injection, a significant reduction in tumor growth was seen in HCT-116-shFTX injected mice. The miR-214-5p inhibitor could abrogate this effect, both in tumor volume and weight (Figure 6A-6C). Furthermore, IHC assays indicated that decreased expression of lncRNA FTX inhibited the expression of Ki67, vimentin, and MMP3, which are markers of cell proliferation, migration, and invasion, respectively (Figure 6D-6F). JAG1 expression decreased in sh-FTX tumors (Figure $6 E, 6 F$ ). Consistent with previous data, the inhibition of miR-214-5p reversed the lncRNA FTX silencing-induced phenomenon in vivo (Figure 6). These findings indicated that lncRNA FTX might play a crucial role in promoting the growth and metastasis of CRC 
in vivo. We demonstrated that lncRNA FTX contributes to CRC growth by sequestering miR-214-5p, which modulates JAG1 expression.

\section{Discussion}

CRC is a devastating disease that has a complicated and somewhat unclear physiopathology. Previous studies have demonstrated critical gene alterations in CRC, but targeting them as therapeutic approaches has not been successful (4). LncRNAs have recently been found to contribute clinically significantly. Some are regarded as diagnostic indicators or prognostic markers for some diseases, including various cancers (27). For instance, lncRNA H19 is regarded as a possible diagnostic parameter in breast cancer patients, and lncRNA D16366 is considered to be a reference marker for predicting the prognosis of hepatocellular carcinoma $(28,29)$. On this basis, our group verified the upregulation of lncRNA FTX expression in CRC and subsequently confirmed the suppression of CRC tumorigenesis by silencing lncRNA FTX. Mechanistically, lncRNA FTX sequester miR-214-5p and therefore releases its repression on JAG1, driving the malignant progression of CRC. The present study's findings provide new evidence that lncRNA FTX is associated with CRC progression and can be considered a potential prognostic biomarker.

LncRNAs have been reported as being related to different cancer types due to the emerging progression of next-generation sequencing, which has increased the number of available cancer transcriptomes (30). Initially, lncRNA FTX was found to be a key factor in malignant progression, dependent on RIG-I targeting to activate PI3K/Akt in hepatocellular carcinoma cells (22), and has since been found to have a regulatory function in other cancers. Zhang et al. revealed that IncRNA FTX could promote the progression and metastasis of gastric cancer through miR-215-3p (18). Liang et al. demonstrated that FTX might select as a novel predictor for the prognostic assessment of glioma patients (31). Furthermore, Huo et al. demonstrated that the cell cytoplasm location of lncRNA FTX contributes to cell proliferation and migration in lung adenocarcinoma by targeting miR-335-5p (23). However, Liu et al. demonstrated that lncRNA FTX inhibits hepatocellular carcinoma malignant progression by binding MCM2 and miR-374a (14). The reason for this inconsistency remains largely unknown. In addition, FTX was found to be expressed at a higher level in female livers than male livers but downregulated in hepatocellular carcinoma tissues in both males and females (14). These findings indicate that FTX plays an important role in tumor progression, although the expression of FTX may have sex differences. However, none have confirmed the role of lncRNA FTX in CRC cells in vivo. Therefore, in the present study, we further revealed the function of lncRNA FTX in vitro and in vivo.

An increasing number of research have demonstrated the interaction between lncRNAs and miRNAs. The association between lncRNAs and miRNAs inhibits the suppressive impact of miRNAs on their target mRNAs. For instance, lncRNA JPX functions as an internal sponge to reduce the expression of miR-33a-5p in lung cancer (32). LncRNA MALAT1, which acts working as a ceRNA, can bind to miR-200c directly and, in endometrioid endometrial carcinoma, decreases its expression (33). Therefore, we conducted a bioinformatics analysis to determine the potential downstream gene(s) of lncRNA FTX. Our findings indicated that lncRNA FTX knockdown upregulated miR214-5p expression, and miR-214-5p attaches to lncRNA FTX in a way specific to the sequence in CRC cells.

Moreover, CRC tissue microarrays in our research showed a negative correlation between lncRNA FTX and miR-214-5p in the same group. lncRNA FTX depletion could suppress the proliferation and migration of CRC cells; the addition of the miR-214-5p inhibitor weakened this inhibition. These results indicated that lncRNA FTX performed its function via miR-214-5p in CRC cells. JAG1 was found to play a vital role in the epithelial to mesenchymal transition of pancreatic cancer cells (34). In the present study, we demonstrated that JAG1 was a direct target of miR-214-5p and regulated CRC progression through the lncRNA FTX-miR-214-5p axis. It is important to note that besides regulation of JAG1, there might be also other targets mediating the tumor suppressor function of lncRNA FTX-miR-214-5p axis. In fact, there are many other target genes of miR-214-5p, such as SOX4 and KLF5, are also important in the metastasis and/or chemoresistance of cancer $(35,36)$. Although we certified that miR-214-5pJAG1 functions as the critical axis regulated by $F T X$, future studies are warranted that investigate the downstream effects of the FTX-miR-214-5p axis on CRC to broaden understanding of how CRC cells evolve and develop.

In conclusion, we identified that lncRNA FTX, which is highly expressed in CRC tissues, could sequester miR214-5p to facilitate CRC progression through the lncRNA $F T X$-miR-214-5p JAG1 regulatory axis. Our research not only explored the downstream regulators of lncRNA FTX 
but also provided new sight into the promising utility of lncRNA FTX as a target of treatment in CRC.

\section{Acknowledgments}

Funding: This study was supported by grants from the National Natural Science Foundation of China (82003220, 82072658 and 81872049), Major logistics research project Foundation (AKJ19J002), Foundation strengthening Program Technical Area Fund projects (2019-JCJQ-JJ-098).

\section{Footnote}

Reporting Checklist: The authors have completed the ARRIVE reporting checklist. Available at https://dx.doi. org/10.21037/atm-21-2755

Data Sharing Statement: Available at https://dx.doi. org/10.21037/atm-21-2755

Conflicts of Interest: All authors have completed the ICMJE uniform disclosure form (available at https://dx.doi. org/10.21037/atm-21-2755). The authors have no conflicts of interest to declare.

Ethical Statement: The authors are accountable for all aspects of the work in ensuring that questions related to the accuracy or integrity of any part of the work are appropriately investigated and resolved. The study was conducted in accordance with the Declaration of Helsinki (as revised in 2013). The study was approved by the Clinical Research Ethics Committee of the Fourth Military Medical University (FMMU) (No.: 202003-183) and informed consent was taken from all individual participants. Animal experiments were performed under a project license (No.: 202003-183) granted by the Institutional Animal Care and Use Committee of FMMU, in compliance with institutional guidelines for the care and use of animals.

Open Access Statement: This is an Open Access article distributed in accordance with the Creative Commons Attribution-NonCommercial-NoDerivs 4.0 International License (CC BY-NC-ND 4.0), which permits the noncommercial replication and distribution of the article with the strict proviso that no changes or edits are made and the original work is properly cited (including links to both the formal publication through the relevant DOI and the license). See: https://creativecommons.org/licenses/by-nc-nd/4.0/.

\section{References}

1. Feng RM, Zong YN, Cao SM, et al. Current cancer situation in China: good or bad news from the 2018 Global Cancer Statistics? Cancer Commun (Lond) 2019;39:22.

2. Dienstmann R, Vermeulen L, Guinney J, et al. Consensus molecular subtypes and the evolution of precision medicine in colorectal cancer. Nat Rev Cancer 2017;17:79-92.

3. Yu T, Guo F, Yu Y, et al. Fusobacterium nucleatum Promotes Chemoresistance to Colorectal Cancer by Modulating Autophagy. Cell 2017;170:548-563.e16.

4. Fearnhead NS, Wilding JL, Bodmer WF. Genetics of colorectal cancer: hereditary aspects and overview of colorectal tumorigenesis. Br Med Bull 2002;64:27-43.

5. Renganathan A, Felley-Bosco E. Long Noncoding RNAs in Cancer and Therapeutic Potential. Adv Exp Med Biol 2017;1008:199-222.

6. Meola N, Pizzo M, Alfano G, et al. The long noncoding RNA Vax2os1 controls the cell cycle progression of photoreceptor progenitors in the mouse retina. RNA 2012;18:111-23.

7. Sigova AA, Mullen AC, Molinie B, et al. Divergent transcription of long noncoding RNA/mRNA gene pairs in embryonic stem cells. Proc Natl Acad Sci U S A 2013;110:2876-81.

8. Kapusta A, Feschotte C. Volatile evolution of long noncoding RNA repertoires: mechanisms and biological implications. Trends Genet 2014;30:439-52.

9. Zhao Y, Xu J, Le VM, et al. EpCAM AptamerFunctionalized Cationic Liposome-Based Nanoparticles Loaded with miR-139-5p for Targeted Therapy in Colorectal Cancer. Mol Pharm 2019;16:4696-710.

10. Wang X, Zhang H, Yang H, et al. Exosome-delivered circRNA promotes glycolysis to induce chemoresistance through the miR-122-PKM2 axis in colorectal cancer. Mol Oncol 2020;14:539-55.

11. Romito A, Rougeulle C. Origin and evolution of the long non-coding genes in the $\mathrm{X}$-inactivation center. Biochimie 2011;93:1935-42.

12. Chureau C, Chantalat S, Romito A, et al. Ftx is a noncoding RNA which affects Xist expression and chromatin structure within the $\mathrm{X}$-inactivation center region. Hum Mol Genet 2011;20:705-18.

13. Furlan G, Gutierrez Hernandez N, Huret C, et al. The Ftx Noncoding Locus Controls X Chromosome Inactivation Independently of Its RNA Products. Mol Cell 2018;70:462-472.e8. 
14. Liu F, Yuan JH, Huang JF, et al. Long noncoding RNA FTX inhibits hepatocellular carcinoma proliferation and metastasis by binding MCM2 and miR-374a. Oncogene 2016;35:5422-34.

15. Zhang W, Bi Y, Li J, et al. Long noncoding RNA FTX is upregulated in gliomas and promotes proliferation and invasion of glioma cells by negatively regulating miR-3423p. Lab Invest 2017;97:447-57.

16. Long B, Li N, Xu XX, et al. Long noncoding RNA FTX regulates cardiomyocyte apoptosis by targeting miR29b-1-5p and Bcl212. Biochem Biophys Res Commun 2018;495:312-8.

17. Yang Y, Zhang J, Chen X, et al. LncRNA FTX sponges miR-215 and inhibits phosphorylation of vimentin for promoting colorectal cancer progression. Gene Ther 2018;25:321-30.

18. Zhang F, Wang XS, Tang B, et al. Long non-coding RNA FTX promotes gastric cancer progression by targeting miR-215. Eur Rev Med Pharmacol Sci 2020;24:3037-48.

19. He X, Sun F, Guo F, et al. Knockdown of Long Noncoding RNA FTX Inhibits Proliferation, Migration, and Invasion in Renal Cell Carcinoma Cells. Oncol Res 2017;25:157-66.

20. Camps J, Nguyen QT, Padilla-Nash HM, et al. Integrative genomics reveals mechanisms of copy number alterations responsible for transcriptional deregulation in colorectal cancer. Genes Chromosomes Cancer 2009;48:1002-17.

21. Mo Y, Wan R, Zhang Q. Application of reverse transcription-PCR and real-time PCR in nanotoxicity research. Methods Mol Biol 2012;926:99-112.

22. Liu Z, Dou C, Yao B, et al. Ftx non coding RNA-derived miR-545 promotes cell proliferation by targeting RIG-I in hepatocellular carcinoma. Oncotarget 2016;7:25350-65.

23. Huo X, Wang H, Huo B, et al. FTX contributes to cell proliferation and migration in lung adenocarcinoma via targeting miR-335-5p/NUCB2 axis. Cancer Cell Int 2020;20:89.

24. Li H, Wang H, Ren Z. MicroRNA-214-5p Inhibits the Invasion and Migration of Hepatocellular Carcinoma Cells by Targeting Wiskott-Aldrich Syndrome Like. Cell Physiol Biochem 2018;46:757-64.

25. Yao Z, Chen Q, Ni Z, et al. Long Non-Coding RNA Differentiation Antagonizing Nonprotein Coding RNA (DANCR) Promotes Proliferation and Invasion of Pancreatic Cancer by Sponging miR-214-5p to Regulate E2F2 Expression. Med Sci Monit 2019;25:4544-52.
26. Shah D, Wyatt D, Baker AT, et al. Inhibition of HER2 Increases JAGGED1-dependent Breast Cancer Stem Cells: Role for Membrane JAGGED1. Clin Cancer Res 2018;24:4566-78.

27. Weng $M, W u ~ D$, Yang $C$, et al. Noncoding RNAs in the development, diagnosis, and prognosis of colorectal cancer. Transl Res 2017;181:108-20.

28. Zhang K, Luo Z, Zhang Y, et al. Circulating lncRNA H19 in plasma as a novel biomarker for breast cancer. Cancer Biomark 2016;17:187-94.

29. Chao Y, Zhou D. IncRNA-D16366 Is a Potential Biomarker for Diagnosis and Prognosis of Hepatocellular Carcinoma. Med Sci Monit 2019;25:6581-6.

30. Huarte $M$. The emerging role of lncRNAs in cancer. Nat Med 2015;21:1253-61.

31. Liang Y, Lu H. Long noncoding RNA FTX is associated with prognosis of glioma patients. J Gene Med 2020;22:e3237.

32. Pan J, Fang S, Tian H, et al. lncRNA JPX/miR-33a-5p/ Twist 1 axis regulates tumorigenesis and metastasis of lung cancer by activating $\mathrm{Wnt} / \beta$-catenin signaling. Mol Cancer 2020;19:9.

33. Li Q, Zhang C, Chen R, et al. Disrupting MALAT1/ miR-200c sponge decreases invasion and migration in endometrioid endometrial carcinoma. Cancer Lett 2016;383:28-40.

34. Cao TH, Ling X, Chen C, et al. Role of miR-214-5p in the migration and invasion of pancreatic cancer cells. Eur Rev Med Pharmacol Sci 2018;22:7214-21.

35. Xie J, Zhu J, Pang J, et al. HLA complex group 11 is involved in colorectal carcinoma cisplatin resistance via the miR-214-5p/SOX4 axis. Oncol Lett 2021;22:535.

36. Pang J, Li Z, Wang G, et al. miR-214-5p targets KLF5 and suppresses proliferation of human hepatocellular carcinoma cells. J Cell Biochem 2018. [Epub ahead of print]. doi: $10.1002 /$ jcb. 27498 .

(English Language Editors: R. Scott and J. Chapnick)

Cite this article as: Pan L, Du M, Liu H, Cheng B, Zhu M, Jia B, Wang Y, He W, Li X, Liu C, Gu J, Li M, Zhang Y, Yao L, Zhang Y, Hao Q. LncRNA FTX promotes the malignant progression of colorectal cancer by regulating the miR-214-5pJAG1 axis. Ann Transl Med 2021;9(17):1369. doi: 10.21037/ atm-21-2755 
A

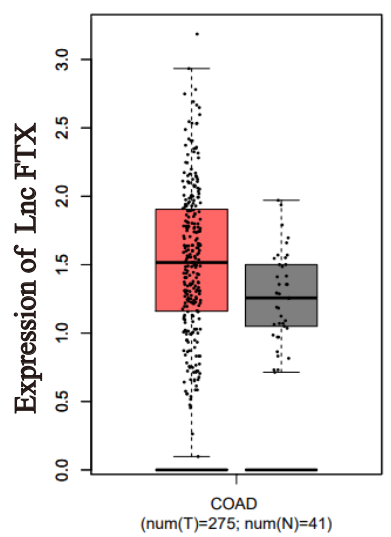

D

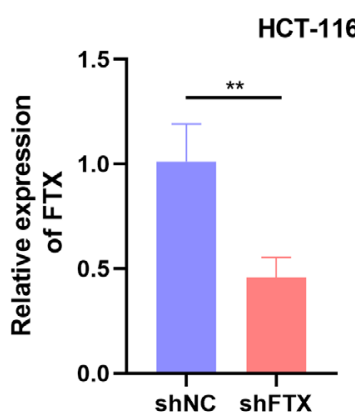

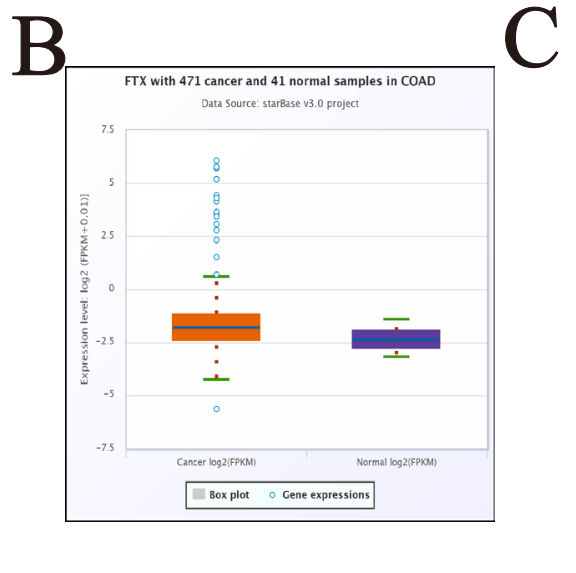

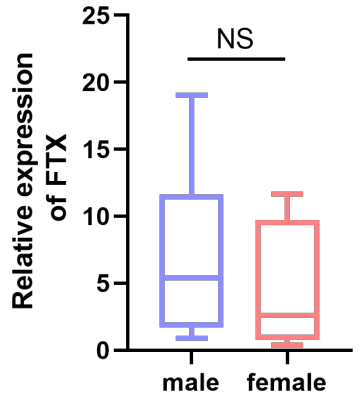

F
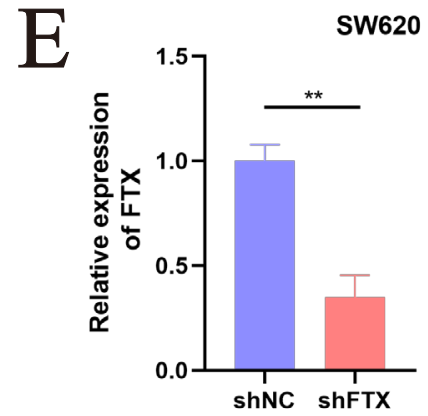

SW620

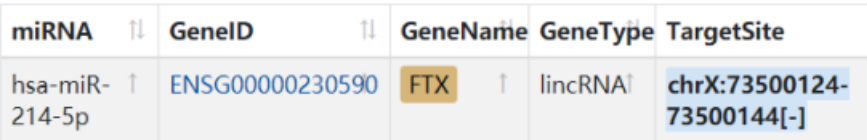
Alignment
Target: $5^{\prime}$ caAGAGCAUAAGU-GACAGGCc $3^{\prime}$
| |||| || |||||||
miRNA : 3' CgUGUCGUUCACAUCUGUCCGu $5^{\text {' }}$
Class
7 mer-
$\mathrm{m} 8$

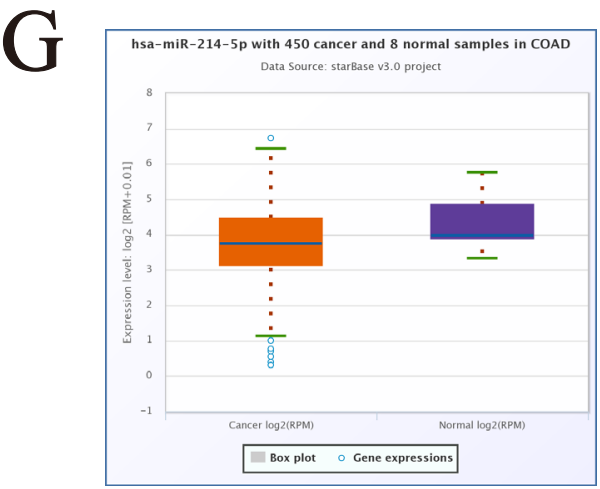

Figure S1 Expression of lncRNA FTX inversely correlates the expression of miR-214-5p in clinical samples and knockdown efficiency of lncRNA FTX in CRC cell lines. (A,B) LncRNA FTX expression in colorectal cancer tissues based on the Gene Expression Profiling Interactive Analysis and TCGA databases. (C) Comparison of lncRNA FTX expression between male and female CRC patients. (D,E) Expression of lncRNA FTX in HCT-116 and SW620 cells following transfection with shNC or sh-FTX was determined by quantitative realtime polymerase chain reaction. (F) Predicted target sites in miR-214-5p and lncRNA FTX based on the starBase database. (G) Expression of miR-214-5p in CRC tissues based on the TCGA database. **, $\mathrm{P}<0.01$; NS, nonsignificant. CRC, colorectal cancer. 